\title{
Functional group resolved nuclear spin relaxation in porous media
}

\author{
Neil Robinson, Eric F. May and Michael L. Johns* \\ Department of Chemical Engineering, University of Western Australia, 35 Stirling Highway, Perth, WA 6009, \\ Australia
}

*Corresponding Author:
Prof Mike Johns
Chair of Chemical and Process Engineering
University of Western Australia

Postal Address:

Department of Chemical Engineering

The University of Western Australia

35 Stirling Highway (M050)

Perth WA 6009

Australia

Phone: +61 (08) 64885664

Email: michael.johns@uwa.edu.au

Fluid Science and Resources Research Group: www.fsr.ecm.uwa.edu.au

\section{Author ORCID:}

Neil Robinson

0000-0002-0893-2190

Eric F. May

0000-0001-5472-6921

Michael L. Johns

0000-0001-7953-0597 


\section{Abstract}

2 Understanding solid-fluid interactions within porous materials is critical for their efficient utilisation across

3 chemical reaction and separation processes. However, detailed characterisation of interfacial phenomena

4 within such systems is hampered by their optically opaque nature. Motivated by the need to bridge this

5 capability gap, we detail here the application of low magnetic field $2 \mathrm{D}{ }^{1} \mathrm{H}$ nuclear spin relaxation

6 measurements as a non-invasive probe of sorbate/sorbent interactions, exploring the relaxation

7 characteristics exhibited by liquid adsorbates confined to a model mesoporous silica. For the first time, we

8 demonstrate the capacity of such measurements to distinguish functional group-specific relaxation

9 phenomena across a diverse range of protic adsorbates of wide importance as solvents, reagents, and 10 hydrogen carriers, with distinct relaxation environments assigned to the alkyl and hydroxyl moieties of the 11 confined liquids. Uniquely, this relaxation behaviour is shown to correlate with adsorbate acidity, with the 12 observed relationship rationalised on the basis of surface-adsorbate proton exchange dynamics. 


\section{Introduction}

2 Characterisation of the solid-liquid interface is critical for the rational development of technologies across the medical, corrosion, environmental and energy sciences. ${ }^{1}$ However, spectroscopic interrogation of the chemical and physical nature of such interfaces remains severely impeded by the need to differentiate the species of interest from the surrounding solid and liquid components. In the case of porous solids - essential to diverse industrial processes such as separations, catalysis, and energy storage - straightforward characterisation of interfacial phenomena is further hindered by the heterogeneous and optically opaque nature of the materials employed, which in turn preclude spectroscopic approaches which necessitate unimpeded access to welldefined surfaces, or require thin adsorbate films. ${ }^{2}$

Nuclear spin relaxation measurements have emerged as a versatile, chemically selective, and non-invasive route for the characterisation of such systems., ${ }^{3,4}$ Utilising appropriate nuclear magnetic resonance (NMR) pulse sequences and hardware this experimental approach measures the longitudinal and/or transverse relaxation behaviour of the spin system under study, as characterised by the time constants $T_{1}$ and $T_{2}$, respectively. While these time constants conform to well-defined relationships with the translational and rotational dynamics of spin-bearing molecules in the unrestricted bulk liquid phase, ${ }^{5}$ the restricted dynamics of fluids within porous media often results in complex relaxation behaviour associated with surface interactions and confinement effects, providing potential insight into both material characteristics and interfacial phenomena. ${ }^{6}$ Such measurements have been employed by the rock physics and petrochemical exploration communities for over five decades, 7,8 providing estimates of oil and gas reservoir quality indicators including pore size distributions, porosity, permeability, and producible hydrocarbon content. ${ }^{9}$ Further wellestablished applications include the observation of cement and plaster hydration kinetics, ${ }^{10,11}$ while emergent fields within the materials chemistry research space now include characterisation of bespoke porous architectures including heterogeneous catalysts, ${ }^{12-14}$ zeolites $^{15}$ and metal-organic frameworks. ${ }^{16}$

The most data-rich methods for elucidating spin relaxation processes in porous media are multidimensional relaxation correlation measurements. ${ }^{17-19}$ Indeed, two-dimensional (2D) relaxation-relaxation $\left(T_{1}-T_{2}{ }^{20-22}\right.$ and $\left.T_{2}-T_{2}{ }^{23,24}\right)$ and diffusion-relaxation $\left(D-T_{2}{ }^{25-28}\right)$ measurements are widely applied to characterise the dynamics of confined fluids, while higher-dimensional experiments, ${ }^{29-32}$ including variants exhibiting spatial resolution, ${ }^{33,34}$ have also been reported. Such measurements are of particular utility in the study of fluids confined to heterogeneous solids as they are essentially independent of the detailed chemical shift phenomena associated with traditional frequency-based NMR methods, wherein spectral peaks are likely to be (i) dominated by species away from the interface of interest, and (ii) unfavourably broadened due to the effects of magnetic susceptibility differences at the solid-liquid interface, ${ }^{35}$ often requiring exotic approaches (see e.g. ref ${ }^{36}$ ) to avoid an intractable loss of spectral resolution. 
1 Here we focus on the interpretation of $2 \mathrm{D}{ }^{1} \mathrm{H}$ (proton) $T_{1}-T_{2}$ relaxation correlation data as a probe of 2 adsorbate identity and interfacial dynamics. Such measurements are particularly advantageous in the 3 presence of multiple fluids, with differences in observed relaxation times - often by an order of magnitude or 4 more - facilitating distinction between components. An established example of this phenomenon is the 5 characterisation of hydrocarbon-bearing shale rocks, ${ }^{37,38}$ wherein the contributions of oil, gas and bituminous 6 hydrocarbons are often readily identified. As first demonstrated by Weber et al., ${ }^{39}$ this concept is also 7 extensible to the observation of competitive displacement processes, wherein the relative populations of 8 correlation peaks associated with different liquids may be followed simultaneously to provide insight into 9 displacement kinetics.

10 In this work, the concept that multiple fluids within the same porous material can present distinct relaxation 11 correlation populations is extended to the realisation and interpretation of relaxation data associated with a 12 single probe fluid exhibiting multiple observable nuclear spin relaxation environments. Specifically, through 13 the exploitation of a series of short-chain alcohols and carboxylic acids imbibed within a model mesoporous 14 silica, we demonstrate for the first time that a wide range of polar-protic adsorbates exhibit discrete proton 15 relaxation characteristics associated with their different hydrogen-bearing moieties. We find that such 16 observations facilitate clear spectroscopic distinction between alkyl and hydroxyl groups without the need for 17 chemical shift analysis. Further motivated by the discovery of a correlation between relaxation characteristics 18 and adsorbate acidity, we provide a clear rationalisation for the observed functional group-specific surface 19 relaxation phenomena in terms of interfacial proton exchange dynamics. 


\section{Nuclear spin relaxation of confined liquids}

3 Figure 1 summarises our experimental approach: Measurements utilised a 2D $T_{1}-T_{2}$ correlation pulse 4 sequence (Figure 1a) implemented on a $12.7 \mathrm{MHz}$ benchtop NMR spectrometer (Figure 1b). Samples comprised a range of short chain liquid hydrocarbons imbibed within a particulate silica material $(4 \mathrm{~mm}$ diameter particles) exhibiting $15 \mathrm{~nm}$ pores (Figure 1c); mesoporous silica was selected as a model porous solid in this work due to its wide-ranging application as a support material in heterogeneous catalysis, and hence relevance to a plethora of interfacial processes. ${ }^{40}$ Further experimental details are provided in Methods.

The $T_{1}-T_{2}$ correlation experiment employed here measures a series of $T_{2}$ relaxation decay processes exhibiting differing degrees of $T_{1}$ encoding (Figure 1d). The resulting 2D relaxation data may then be inverted ${ }^{41}$ to provide a 2D $T_{1}-T_{2}$ population distribution, wherein correlation peaks provide insight into the relative probability of the system exhibiting a given combination of $T_{1}$ and $T_{2}$ relaxation times. Figures $\mathbf{1 e}$ and $\mathbf{1 f}$ show example correlation data for imbibed cyclohexane and acetone, explored here as archetypal apolar and polaraprotic adsorbates, respectively. Any liquid outside of the porous material was removed before analysis (see Methods) such that all observed relaxation characteristics are associated with confined species. A single correlation peak is observed in each case, characteristic of imbibed species exhibiting a single effective ${ }^{1} \mathrm{H}$ relaxation environment as a result of fast-exchange between adsorbed species and bulk-like molecules away from the solid-liquid interface. ${ }^{42,43}$ Under such conditions, and assuming surface-limited relaxation, ${ }^{6}$ a wellestablished general expression for the observed relaxation rates $T_{i, o b s}^{-1}$ (with $i \in\{1,2\}$ ) is known: ${ }^{17}$

$$
\frac{1}{T_{i, o b s}} \approx \frac{1}{T_{i, b u l k}}+\frac{S}{V} \frac{\delta}{T_{i, \text { surf }}}
$$

Here $S / V$ is the surface-to-volume ratio of the confining pore structure, $\delta$ the length scale of the adsorbed surface layer, $T_{i, \text { surf }}$ the relaxation time constants of the adsorbed population, and $T_{i, b u l k}$ the time constants of the unrestricted fluid (see Figure 1c). This expression exemplifies the inherent sensitivity of nuclear spin relaxation measurements to both material properties $(S / V$; providing sensitivity to pore size) and interfacial chemistry $\left(\delta / T_{i, s u r f}\right.$; commonly termed the surface relaxivity). Moreover, given the general case that $T_{i, s u r f} \ll T_{i, b u l k}$, the dimensionless ratio of observed relaxation time constants can be reduced to to

$T_{1, \text { obs }} / T_{2, \text { obs }} \sim T_{1, \text { surf }} / T_{2, \text { surf }} .{ }^{44}$ This surface ratio $T_{1, \text { surf }} / T_{2, \text { surf }}$ is sensitive to the activated translational dynamics of molecules at the solid-liquid interface, ${ }^{45}$ and is considered a non-invasive probe of surface affinity $^{21,46}$ (additional surface relaxation theory is detailed in Supplementary Note 1). As the experimentally accessible ratio $T_{1, o b s} / T_{2, o b s}$ is largely unaffected by the terms $\delta$ and $S / V$ in Equation (1), acquisition of this metric provides a novel route for the comparison of interfacial phenomena either between different porous 
1 media imbibed with the same liquid, or as demonstrated here, multiple liquids within the same porous material.

3 As the ill-posed nature of the inversion process required to obtain the $2 \mathrm{D}$ relaxation distributions (an inverse 4 Laplace transform ${ }^{47}$ ) is highly susceptible to the influence of experimental noise, ${ }^{41}$ we restrict our analysis of 5 such distributions to the modal relaxation characteristics of each correlation peak observed, making no 6 attempt to infer insight from correlation peak shapes. The modal $T_{1, o b s} / T_{2, o b s}$ ratios of the correlation peak 7 within Figures $1 \mathbf{e}$ and $\mathbf{1 f}$ (from here on in referred to as $\left\langle T_{1} / T_{2}\right\rangle$ ) are indicated by a dashed diagonal lines, with 8 the larger $\left\langle T_{1} / T_{2}\right\rangle$ value of acetone $\left(\left\langle T_{1} / T_{2}\right\rangle=1.47 \pm 0.01\right)$, compared to cyclohexane $\left(\left\langle T_{1} / T_{2}\right\rangle=1.13 \pm\right.$ $90.02)$ providing a clear demonstration of the increased affinity of polar adsorbates for oxide pore surfaces, 10 relative to apolar alkanes. ${ }^{48}$ 

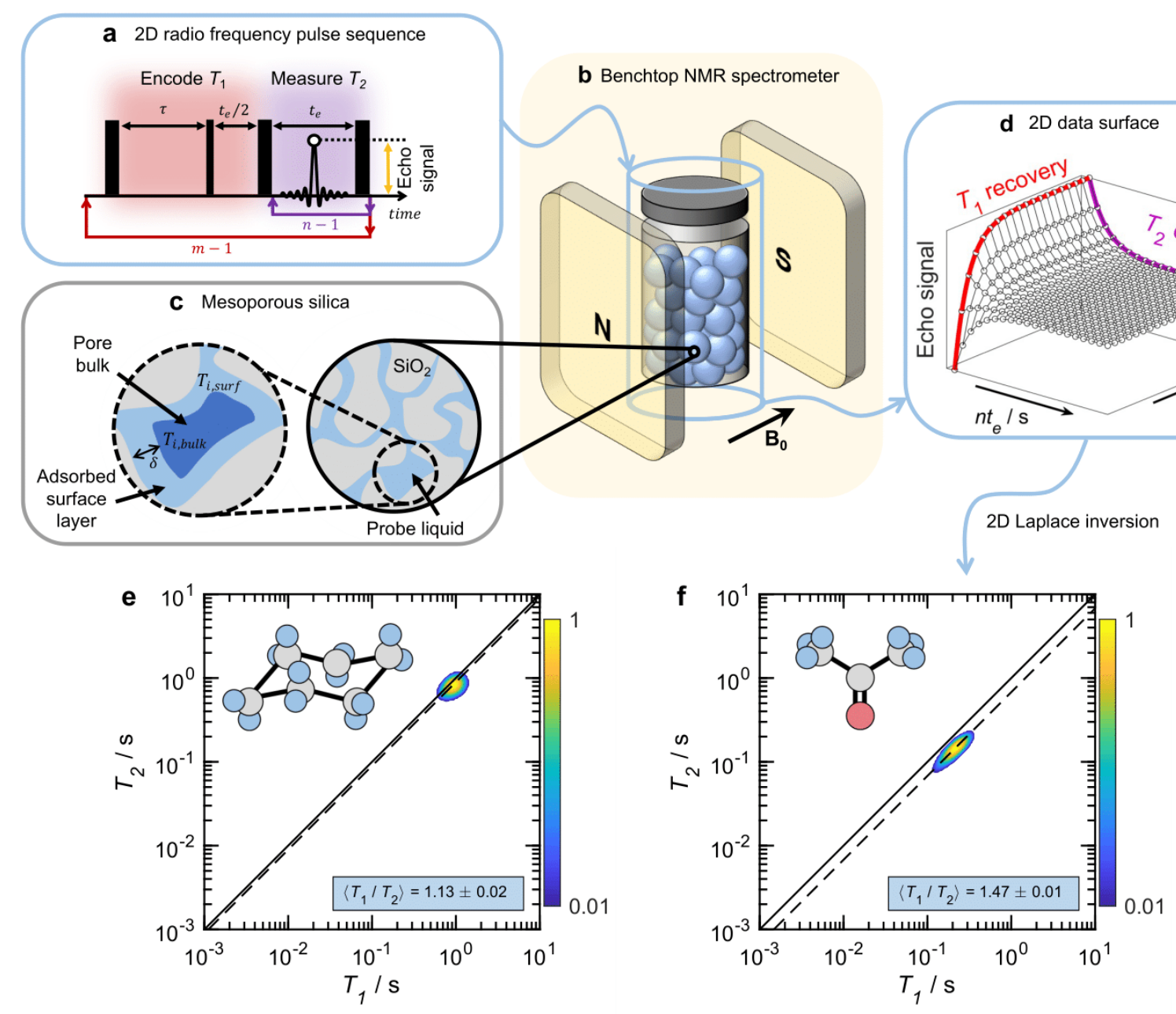

2 Figure 1. Experimental approach. Measurements employed (a) a 2D $T_{1}-T_{2}$ pulse sequence and (b) a low-

3 field benchtop NMR spectrometer with a $B_{0}=0.3 \mathrm{~T}\left(v_{0}\left\{{ }^{1} \mathrm{H}\right\}=12.7 \mathrm{MHz}\right)$ parallel plate magnet array. The porous

4 silica $\left(\mathrm{SiO}_{2}\right)$ material comprised $4 \mathrm{~mm}$ diameter spheres with $15 \mathrm{~nm}$ pores ( $\mathbf{b}$ and $\mathbf{c}$ ), and was soaked in each

5 probe liquid for 48 hours, with the inter-particle liquid then removed. By cycling the 2D pulse sequence in (a)

6 through $m$ different $\tau$ values and recording the intensity of the resulting $n$ echoes a $\left(n t_{e} \times m \tau\right)$ data surface

7 is generated (d), which may be inverted to generate a 2D probability distribution of $T_{1}$ and $T_{2}$ times (see

8 Methods). Example ${ }^{1} \mathrm{H} T_{1}-T_{2}$ correlation data for cyclohexane and acetone within this silica material are

9 shown in $\mathbf{e}$ and $\mathbf{f}$, respectively. The magnitude of each correlation peak indicates the relative probability of

10 each system exhibiting a particular combination of $T_{1}$ and $T_{2}$ relaxation times, as indicated by the colour bars.

11 Solid diagonal lines indicate the parity ratio $T_{1} / T_{2}=1$, while the modal relaxation time ratio $\left\langle T_{1} / T_{2}\right\rangle$ of each 12 correlation peak is indicated by dashed diagonal lines; $\left\langle T_{1} / T_{2}\right\rangle$ values are specified in each case. The molecular 13 structure of each adsorbate is also given: $\mathrm{C}, \mathrm{O}$ and $\mathrm{H}$ atoms are coloured grey, red and blue, respectively. 
2 Figure 2 shows ${ }^{1} \mathrm{H} T_{1}-T_{2}$ distributions for a series of small polar-protic hydrocarbons within the same silica material; the example adsorbates studied here include short-chain primary alcohols (methanol, ethanol, 1propanol and 1-butanol), secondary alcohols (2-propanol and 2-butanol) and simple carboxylic acids (acetic acid, propanoic acid and butanoic acid). In contrast to Figure 1, two distinct correlation peaks are clearly apparent within each data set, exhibiting significantly different modal $T_{1}$ and $T_{2}$ times $\left\langle\left\langle T_{1}\right\rangle\right.$ and $\left\langle T_{2}\right\rangle$, respectively; values are provided in Supplementary Table 1). Any inter-particle liquid was again removed from these samples, such that all observed relaxation populations must arise from the confined fluids within the silica pore network. We note these peaks cannot be assigned to the presence of multiple pore environments with varying surface-to-volume ratios (see Equation (1)), which, given the similar self-diffusion properties of short-chain alcohols and acids to our aprotic adsorbates (demonstrated elsewhere ${ }^{49-51}$ ) would also be apparent within the cyclohexane and acetone data in Figure 1. Proton-proton scalar coupling artefacts ${ }^{52}$ may also be discounted due to the very short echo time employed in these measurements (see Methods). Rather, these populations are assigned to the distinct relaxation characteristics of the alkyl $\left(\mathrm{C}_{x}{ }^{1} \mathrm{H}_{y}\right)$ and hydroxyl $\left(-\mathrm{O}^{1} \mathrm{H}\right)$ proton environments of each adsorbate. Our assignments are supported by previous spectrally-resolved (highfield) $T_{1}$ measurements of methanol within a range of porous oxide materials, ${ }^{53}$ which revealed the hydroxyl proton to exhibit distinctly more rapid rates of $T_{1}$ relaxation than those within the alkyl environment. Correlation peaks at short $\left\langle T_{1}\right\rangle$ and $\left\langle T_{2}\right\rangle$ values are therefore assigned to the hydroxyl ${ }^{1} \mathrm{H}$ environments of each adsorbate, while peaks at longer relaxation times are assigned to the corresponding alkyl environments. These assignments are further consistent with the integrated peak ratios obtained from each correlation plot, which we expect to reflect the ratio of protons within each chemical environment; these ratios are correlated against the expected alkyl/hydroxyl ratio of each adsorbate in Figure 3, demonstrating a strong, positive correlation. Given the complex surface exchange phenomena described and discussed below, however, we note that a 1:1 parity between observed and expected alkyl/hydroxyl ratios is not expected for these data.

While we recognise previous analyses of confined methanol suggest the existence of two distinct relaxation populations, ${ }^{21,54-56}$ the correlation data in Figure 2 exemplifies the first observation of such phenomena in longer chain primary alcohols, as well as the first such data for both secondary alcohols and carboxylic acids. While high and intermediate field measurements have failed to evidence the existence of multiple relaxation environments in the presence of longer chain $\left(>C_{1}\right)$ liquid hydrocarbons, ${ }^{21,56}$ we attribute the clear and persistent observation of hydroxyl group relaxation phenomena across our range of polar-protic adsorbates to the combination of short experimental echo times and low magnetic field strength (see Methods); these experimental conditions significantly reduce the impact of magnetic susceptibility contrast effects on the measurement of short $T_{2}$ times, ${ }^{35}$ facilitating the accurate measurement of relaxation data associated with rapidly relaxing hydroxyl protons. Collectively, the correlation data detailed within Figure $\mathbf{2}$ confirms, for the 
1 first time, the widespread existence of functional group-specific relaxation phenomena across a broad range

2 of organic molecules of importance as solvents, reagents, and hydrogen carriers. In the remainder of this work

3 we identify and examine distinct trends in the observable relaxation characteristics presented by these 4 adsorbates. 

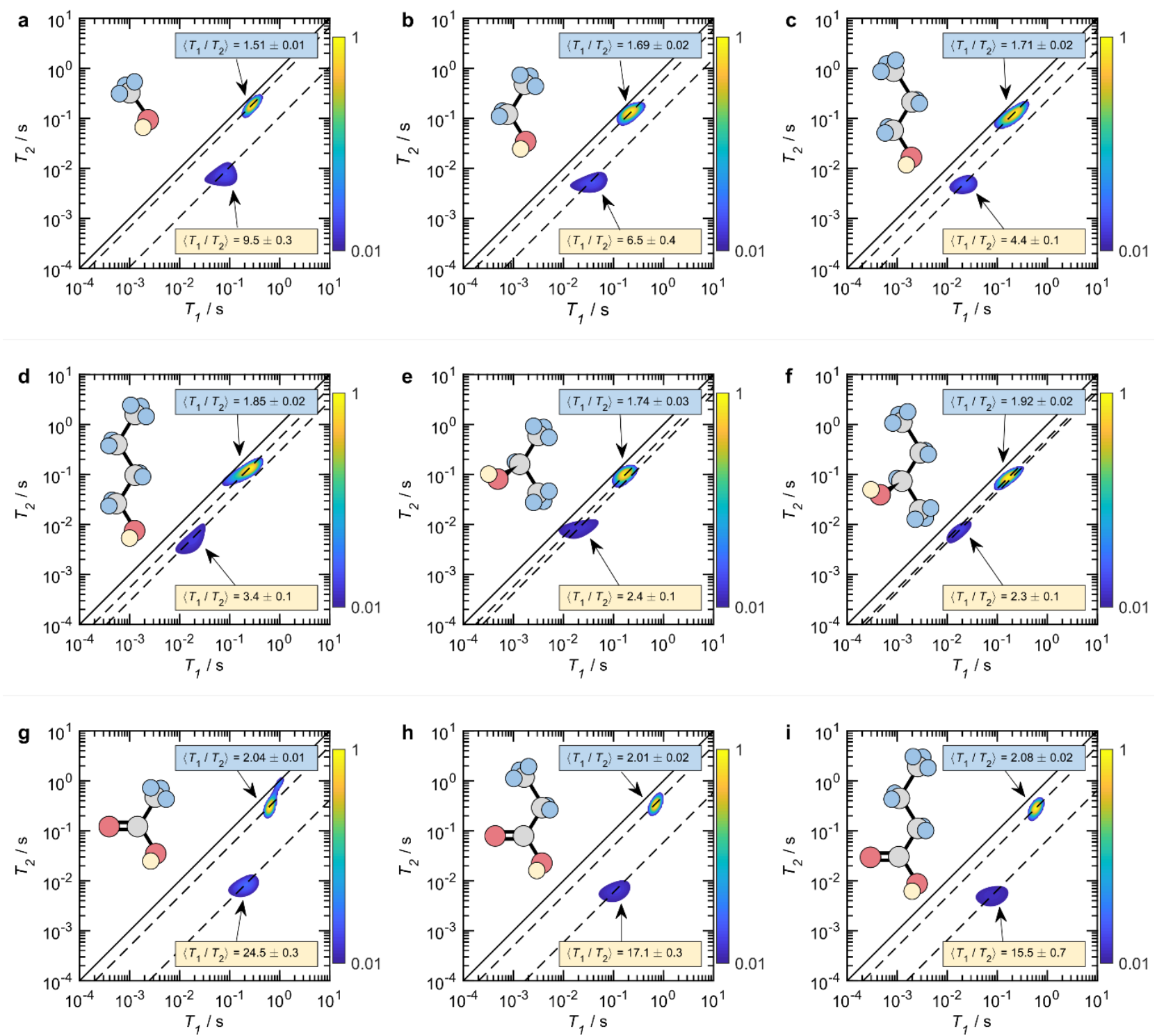

2 Figure 2. Relaxation characteristics of protic adsorbates. ${ }^{1} \mathrm{H} T_{1}-T_{2}$ correlation data for a - d primary 3 alcohols (a methanol, $\mathbf{b}$ ethanol, c 1-propanol and $\mathbf{d}$ 1-butanol), $\mathbf{e}-\mathbf{f}$ secondary alcohols (e 2-propanol and $\mathbf{f}$ 4 2-butanol), and $\mathbf{g}-\mathbf{i}$ carboxylic acids ( $\mathbf{g}$ acetic acid, $\mathbf{h}$ propanoic acid and $\mathbf{i}$ butanoic acid) in mesoporous silica 5 (exhibiting $15 \mathrm{~nm}$ pores) at $12.7 \mathrm{MHz}$. The magnitude of each correlation peak indicates the relative probability 6 of each system exhibiting a particular combination of $T_{1}$ and $T_{2}$ relaxation times, as indicated by the colour bars. Solid diagonal lines indicate the parity ratio $T_{1} / T_{2}=1$, while the modal relaxation time ratio $\left\langle T_{1} / T_{2}\right\rangle$ of each correlation peak is indicated by dashed diagonal lines; $\left\langle T_{1} / T_{2}\right\rangle$ values are specified in each figure. The molecular structure of each adsorbate is also given: $\mathrm{C}$ and $\mathrm{O}$ atoms are coloured grey and red, respectively. Aprotic $\mathrm{H}$ are coloured blue while protic $\mathrm{H}$ are shown in yellow. Correlation peaks at long and short $T_{2}$ are assigned to aprotic and protic ${ }^{1} \mathrm{H}$-containing moieties, respectively. 


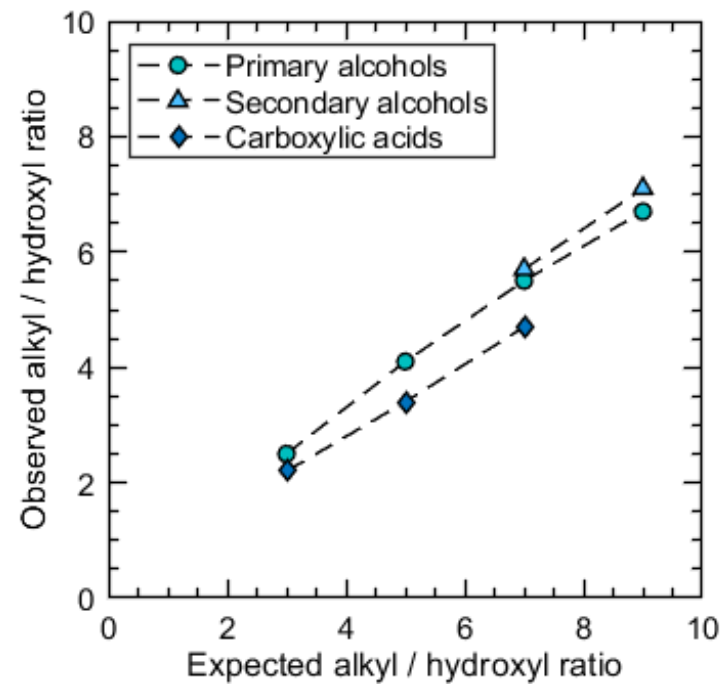

2 Figure 3. Correlation peak integrals. Integrated peak ratios from the correlation data presented in Figure 2 as 3 a function of the expected alkyl/hydroxyl ratio of the same adsorbates. 
2 Figure 4a provides a summary of the $\left\langle T_{1} / T_{2}\right\rangle$ values obtained from the correlation data presented in Figure 2.

3 A general increase in the alkyl $\left\langle T_{1} / T_{2}\right\rangle$ of alcohols is evident with increasing carbon chain length, from

$4\left\langle T_{1} / T_{2}\right\rangle=1.51 \pm 0.01$ for methanol to $\left\langle T_{1} / T_{2}\right\rangle=1.85 \pm 0.02$ for 1 -butanol, and from $\left\langle T_{1} / T_{2}\right\rangle=1.74 \pm$

50.08 for 2-propanol to $\left\langle T_{1} / T_{2}\right\rangle=1.92 \pm 0.02$ for 2-butanol. These observations regards the alkyl $\left\langle T_{1} / T_{2}\right\rangle$

6 ratio are consistent with previous investigations of short-chain primary alcohol behaviour in porous silica ${ }^{21}$

7 and alumina, ${ }^{56}$ with the measured relaxation time ratios found to correlate with adsorbate surface affinity. ${ }^{21}$

8 Conversely, our hydroxyl $\left\langle T_{1} / T_{2}\right\rangle$ values demonstrate a clear decrease with increasing carbon chain length,

9 with this decrease noticeably evident across both the primary alcohol and carboxylic acid data sets. To enable

10 further discussion of this discrepancy we introduce a new metric of the form $\Delta\left\langle T_{1} / T_{2}\right\rangle=\left\langle T_{1} / T_{2}\right\rangle_{\text {hydroxyl }}$

$11\left\langle T_{1} / T_{2}\right\rangle_{\text {alkyl }}$; this metric quantifies the difference in $\left\langle T_{1} / T_{2}\right\rangle$ between the alkyl and hydroxyl relaxation

12 populations of each adsorbate and is independent of their absolute values (see Figure 4a), which we expect

13 to scale with molecular surface affinity. ${ }^{21}$ The overall progression of $\Delta\left\langle T_{1} / T_{2}\right\rangle$ is: carboxylic acids $\gg$ primary

14 alcohols $>$ secondary alcohols (see Supplementary Table 1 for values), with values further decreasing as a function of increased carbon chain length in each case. Given these behaviours mirror the well-known trends in the acidity of these adsorbates, we conjecture that a comprehensive understanding of our observed relaxation data likely requires consideration of hydroxyl proton dissociation. This interpretation is supported in Figure $\mathbf{4} \mathbf{b}$ which details a plot of $\Delta\left\langle T_{1} / T_{2}\right\rangle$ against adsorbate $p K_{a}$, employed here as a convenient metric of liquid-phase acidity. A strong correlation is clearly apparent between increasing $\Delta\left\langle T_{1} / T_{2}\right\rangle$ and decreasing $p K_{a}$ (indicative of increased acidity).

We rationalise the above trend through recognition of the dominant surface interaction mechanism between (hydroxylated) silica surfaces and polar protic molecules. Such interactions are governed by surface-adsorbate hydrogen bonding interactions, which provide not only a favourable bonding mode between protic adsorbates and surface-bound hydroxyl groups, ${ }^{57,58}$ but also a well-established means for proton exchange. ${ }^{59-61}$ Exchange interactions during the encoding periods of $T_{1}-T_{2}$ correlation measurements have significant potential to bias the resulting relaxation characteristics. This concept is elucidated by considering two coupled magnetisation reservoirs, $M_{s o l}$ and $M_{a d s}$, which we associate with solid-bound and adsorbate-bound hydroxyl protons, respectively. Relaxation during the $T_{1}$ and $T_{2}$ encoding periods of the pulse sequence in Figure 1a is given by: ${ }^{62}$

$$
\frac{d}{d t}\left[\begin{array}{l}
M_{\text {sol }} \\
M_{\text {ads }}
\end{array}\right]=\left[\begin{array}{cc}
-T_{1, \text { sol }}^{-1}-k_{1} & k_{2} \\
k_{1} & -T_{1, a d s}^{-1}-k_{2}
\end{array}\right]\left[\begin{array}{c}
M_{\text {sol }}-M_{\text {sol }}^{0} \\
M_{a d s}-M_{a d s}^{0}
\end{array}\right]
$$




$$
\frac{d}{d t}\left[\begin{array}{l}
M_{s o l} \\
M_{a d s}
\end{array}\right]=\left[\begin{array}{cc}
-T_{2, s o l}^{-1}-k_{1} & k_{2} \\
k_{1} & -T_{2, a d s}^{-1}-k_{2}
\end{array}\right]\left[\begin{array}{l}
M_{s o l} \\
M_{a d s}
\end{array}\right]
$$

1 respectively, where $T_{i, s o l}$ and $T_{i, a d s}$ (with $i \in\{1,2\}$ ) are the relaxation time constants of the two reservoirs.

2 The terms $M_{a d s}^{0}$ and $M_{\text {sol }}^{0}$ are the equilibrium magnetisations of the two reservoirs, which are directly 3 proportional to the intrinsic number of protons within each environment. The exchange rate constants $k_{1}$ and $4 \quad k_{2}$ then quantify the rates of proton migration between surface and adsorbate hydroxyl groups, with the overall exchange rate $k=k_{1}+k_{2}$. Simple solutions to these expressions emerge in the limits of and slow $6\left(k \ll\left|T_{2, \text { sol }}^{-1}-T_{2, a d s}^{-1}\right|\right)$ and fast $\left(k \gg\left|T_{1, \text { sol }}^{-1}-T_{1, \text { ads }}^{-1}\right|\right)$ exchange, giving rise to either two distinctly separate relaxation populations or a single, highly averaged population, respectively. ${ }^{63}$ In the intermediate regime between these limiting cases the observed relaxation characteristics are sensitive to the relative magnitudes of the inherent relaxation, exchange rates and equilibrium magnetisations; upon approaching the fast exchange limit a single population is expected, exhibiting relaxation times sensitive to $k^{64}$

11 Simple detection of the exchange regime relevant to our data is obviated by the lack of observable surface 12 hydroxyl populations, ${ }^{37,65}$ with the true relaxation behaviour of our experimental systems further complicated by adsorption/desorption processes. However, given the high sensitivity of the ratio $T_{1} / T_{2}$ to the adsorbed surfaced layer (see Supplementary Note 1), we conjecture that rapid exchange of this form provides a significant contribution to the hydroxyl group $\left\langle T_{1} / T_{2}\right\rangle$ behaviour observed in Figure 2. As solid-state structures exhibit long $T_{1}$ and short $T_{2}$ values, ${ }^{5}$ surface-adsorbate proton exchange dynamics occurring near the fastexchange limit are expected to decrease the observed hydroxyl $T_{2}$ times, while propagating a corresponding increase in $T_{1}$. An increase in exchange rate is therefore expected to increase $\Delta\left\langle T_{1} / T_{2}\right\rangle$ as hydroxyl group exchange dynamics progressively increase the associated $\left\langle T_{1} / T_{2}\right\rangle$ values away from that of the nonexchanging alkyl environment.

Considering the trend demonstrated in Figure $\mathbf{4 b}$, the increase in $\Delta\left\langle T_{1} / T_{2}\right\rangle$ anticipated to arise from such dynamics as a function of increased exchange rate clearly suggests that more acidic adsorbates undergo more rapid rates of proton dissociation at the solid/liquid interface. Despite the typical disparity between the thermodynamic and kinetic contributions to exchange phenomena, an approximate relationship between hydroxyl exchange rates and adsorbate acidity is obtained by considering the double-well potential energy surface in Figure 4c. Assuming classical dynamics (i.e. neglecting tunnelling) the exchange rates between free energy minima is given by the Eyring equation: $k_{i}=\left(k_{B} T / h\right) \exp \left(-\Delta^{\ddagger} G_{i} / R T\right)$ (with $\left.i \in\{1,2\}\right)$, where $k_{B}, h$ and $R$ are the Boltzmann constant, Planck constant and gas constant, respectively, $T$ is the absolute temperature, and $\Delta^{\ddagger} G_{i}$ are the activation free energy barrier heights. Thermodynamically, the free energy change for a given proton dissociation process is $\Delta G \approx 2.303 R T p K_{a}$. As proton exchange between potential wells is considered a pair of coupled pseudo-bimolecular reactions, ${ }^{66}$ the overall difference in hydrogen bond 
potential well depth is determined by the difference in $p K_{a}$ values between the two protonated states,

$2 \Delta p K_{a} \cdot{ }^{67}$ These concepts are readily translatable to the consideration of proton exchange dynamics at solidliquid interfaces. Crucially, for the purpose of rationalising the observed trend between relaxation

4 characteristics and adsorbate $p K_{a}$ detailed in Figure $\mathbf{4 b}$, we recognise that for the comparison of exchange processes between a single silica material and multiple distinct adsorbates, changes in $\Delta p K_{a}$ may be assumed dependent on only the adsorbate $p K_{a}$. With the free energy minimum associated with the solid surface essentially fixed across the range of experiments performed, adsorbate $p K_{a}$ will therefore indirectly dictate the free energy barrier height, in turn influencing observable proton exchange rates $k=k_{1}+k_{2}$. We therefore consider adsorbate $p K_{a}$ an approximate and indirect indicator of overall hydroxyl group exchange rates, in turn providing a generalised rationale for the correlation observed in Figure $\mathbf{4} \mathbf{b}$. This reasoning is further consistent with the peak integral ratio data presented in Figure 3, wherein the observed alkyl/hydroxyl ratios of our adsorbates are clearly reduced from their expected values in order of increasing acidity; adsorbates undergoing significant surface-adsorbate proton exchange will present larger hydroxyl relaxation populations than expected, hence reducing the experimentally observed alkyl/hydroxyl ratio.

To support the above interpretation, the ability of our model silica material to undergo proton exchange interactions with adsorbed hydrocarbons was confirmed via deuterium exchange experiments, with methanol-d4 $\left(\mathrm{CD}_{3} \mathrm{OD} ; \mathrm{D} \equiv{ }^{2} \mathrm{H}\right)$ utilised as a probe fluid. The salient features of the expected solid-liquid interactions are shown in Figure 5a. In the absence of interfacial proton exchange the methanol-d4 probe will remain invisible to ${ }^{1} \mathrm{H}$ NMR analysis; if exchange occurs, however, we expect a growth in detectable ${ }^{1} \mathrm{H}$ NMR signal as surface proton scrambling facilitates the formation of $\mathrm{CD}_{3} \mathrm{OH}$. Figure $\mathbf{5 b}$ compares ${ }^{1} \mathrm{H} T_{2}$ decay data from methanol-d4 (nominal purity $=99.8 \%$ ) with that from a sample of mesoporous silica exposed to excess methanol-d4 for 24 hours; unlike the samples prepared for 2D $T_{1}-T_{2}$ analysis, this system therefore comprises both free and confined liquid. While signal arising from the neat sample (bulk methanol-d4) lies within the spectrometer noise floor, the system comprising mesoporous silica in excess methanol-d4 clearly exhibits an observable $T_{2}$ decay signal, confirming the presence of $N M R$ active $C_{3} O H$, and hence the occurrence of surface-adsorbate proton exchange interactions. Supplementary Note $\mathbf{2}$ further explores the consistent temporal increase of this detectable signal over the first 24 hours. 

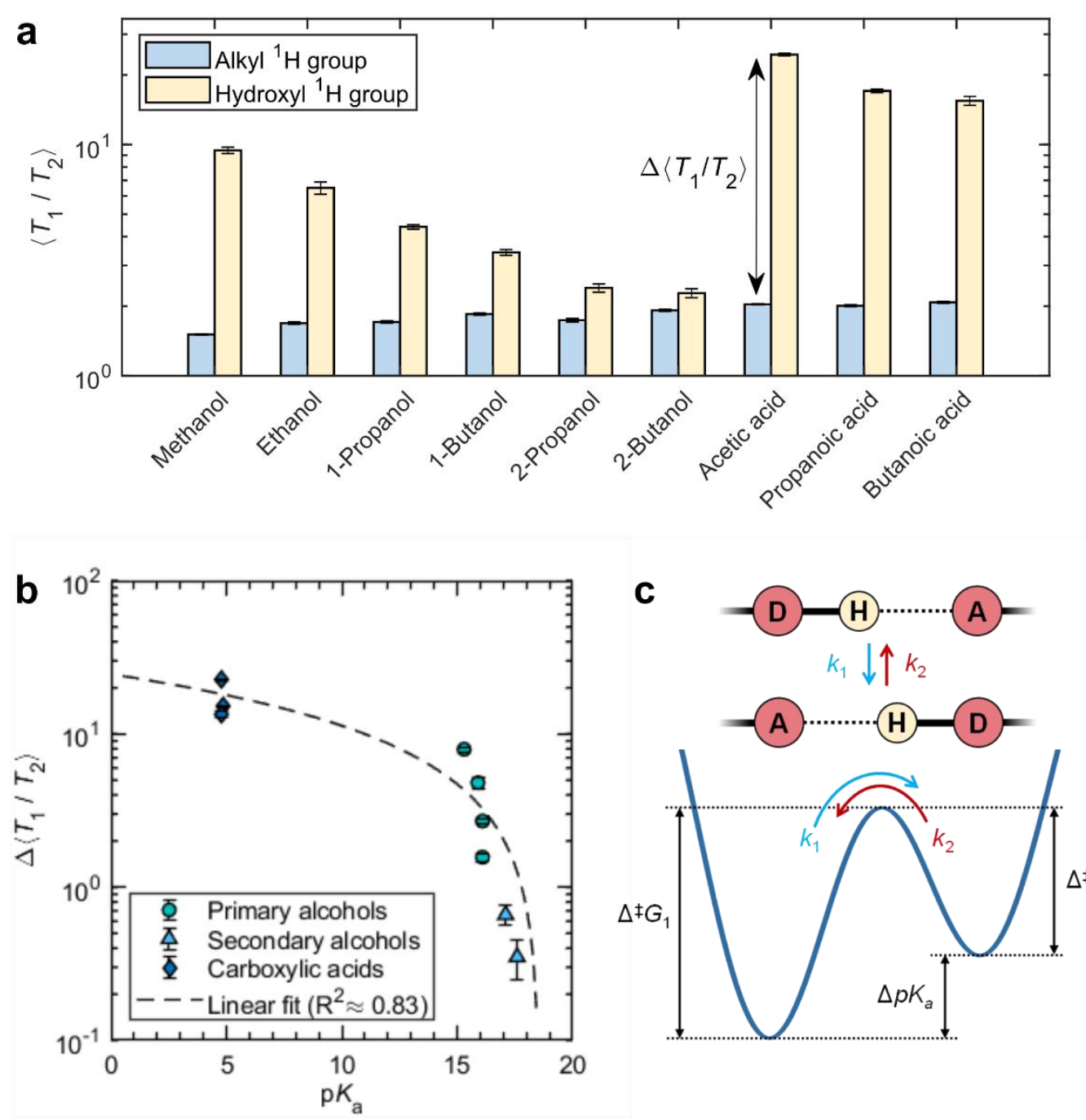

C
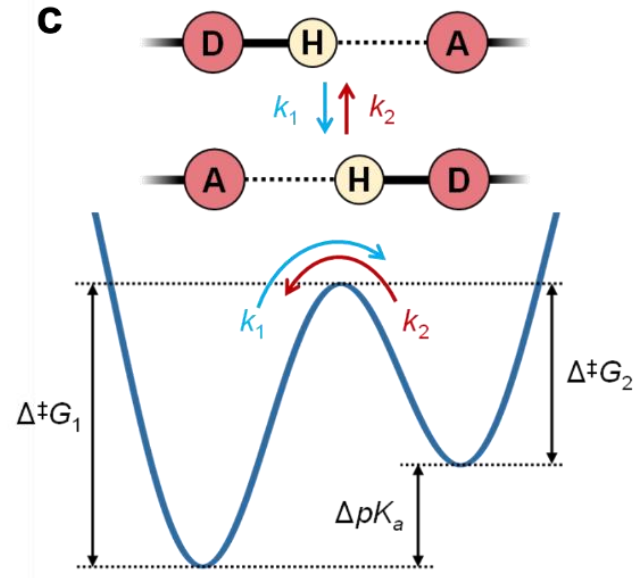

2 Figure 4. Relaxation trends of protic adsorbates. a Summary of modal alky and hydroxyl ${ }^{1} \mathrm{H}$ relaxation time 3 ratios $\left\langle T_{1} / T_{2}\right\rangle$; values correspond with the dashed diagonal lines in Figure 2. b Correlation between the 4 difference in alkyl and hydroxyl relaxation time ratios $\Delta\left\langle T_{1} / T_{2}\right\rangle$ and adsorbate $\mathrm{p} K_{\mathrm{a}}$, including a linear fit. c 5 Double-well potential energy surface for proton exchange between hydrogen bond donors (D) and acceptors 6 (A). The forward and back exchange rates $k_{1}$ and $k_{2}$ are hence determined by the activation free energies $7 \quad \Delta^{\ddagger} G_{1}$ and $\Delta^{\ddagger} G_{2}$, respectively, while the difference in potential well depth is determined by the difference in $8 \quad p K_{a}$ of the two protonated states, $\Delta p K_{a}$. 
a

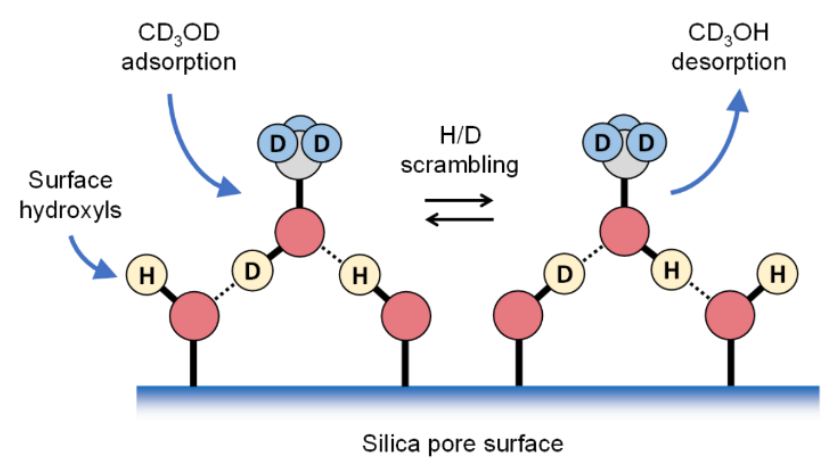

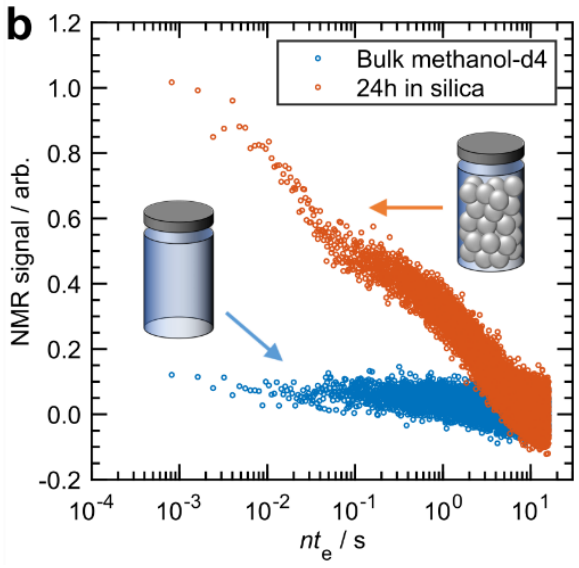

2 Figure 5. Proton scrambling at the methanol/silica interface. a Expected interactions between methanol-d4 $3 \quad\left(\mathrm{CD}_{3} \mathrm{OD}\right)$ and silica pore surfaces. ${ }^{1} \mathrm{H}$ NMR-invisible $\mathrm{CD}_{3} \mathrm{OD}$ molecules adsorb via hydrogen bonding interactions 4 with surface hydroxyl groups, facilitating $\mathrm{H} / \mathrm{D}$ scrambling. Desorbing $\mathrm{CD}_{3} \mathrm{OH}$ will then be visible to ${ }^{1} \mathrm{H} N M R$ 5 analysis. b CPMG $T_{2}$ decay data for bulk $\mathrm{CD}_{3} \mathrm{OD}$ (blue) and $\mathrm{CD}_{3} \mathrm{OD}$ combined in excess with dry mesoporous 6 silica for 24 hours (orange). The clear ${ }^{1} \mathrm{H} T_{2}$ decay signal in the case of $\mathrm{CD}_{3} \mathrm{OD}$ imbibition within silica confirms 7 surface $\mathrm{H} / \mathrm{D}$ scrambling and the formation of ${ }^{1} \mathrm{H}$ NMR-active $\mathrm{CD}_{3} \mathrm{OH}$. 


\section{Conclusions}

2 The identification of functional group-specific nuclear spin relaxation across the diverse range of adsorbates

3 examined here is of clear significance for the accurate interpretation of NMR relaxation data acquired from

4 systems involving polar-protic probe fluids and hydroxylated pore surfaces. The example solid/liquid systems

5 explored here are of particular relevance to the interrogation of solvent effects in liquid-phase heterogeneous

6 catalysis, wherein the utilisation of nuclear spin relaxation measurements to infer interfacial phenomena is

7 now a rapidly evolving field. Furthermore, the unambiguous assignment of multiple relaxation populations to

8 individual adsorbates undergoing surface-adsorbate proton exchange is expected to better inform the

9 application and interpretation of multidimensional relaxation measurements when applied to a range of

10 complex porous systems, averting the erroneous interpretation of correlation data in terms of material

11 structures or the presence of multiple adsorbates. Although not explored here, we note that the clear

12 distinction between alkyl and hydroxyl relaxation populations along the $T_{2}$ dimension of our $2 \mathrm{D}$ correlation

13 data suggests functional group-specific insight is also readily accessible via rapid one-dimensional $T_{2}$

14 measurements, facilitating the extension of such analyses to temporally resolved experimental systems; this

15 concept is of particular relevance to the study of competitive displacement dynamics, which we aim to pursue

16 in future work. Finally, we note that the clear correlation between nuclear spin relaxation characteristics and

17 adsorbate acidity strongly motivates further investigation into how relaxation phenomena may be exploited

18 as a robust and non-invasive probe of adsorbate identity and/or material surface chemistry within a diverse

19 range of porous architectures. 
Methods

\section{Materials and sample preparation}

3 A commercial Q15 mesoporous silica gel material (4 mm diameter spherical particles; nominal mean pore size:

$4 \quad 15 \mathrm{~nm}$ ) was obtained from Fuji Silysia Chemical Ltd. (Japan). The material was first refluxed in deionised water

5 (obtained onsite at the Australian Resources Research Centre, Perth, Australia) at $120{ }^{\circ} \mathrm{C}$ for 4 hours to

6 hydroxylate the pore surfaces. The Q15 was then dried in air at $110^{\circ} \mathrm{C}$ for 12 hours, and for an additional 3 hours at $110^{\circ} \mathrm{C}$ under vacuum $\left(10^{-3}\right.$ bar $)$.

8 Cyclohexane (Thermofisher Scientific, >99\%), acetone (ChemSupply Australia, >98\%), methanol (ChemSupply 9 Australia, >99.9\%), ethanol (ChemSupply Australia, >99.9\%), 1-propanol (ChemSupply Australia, >99.8\%), 2 propanol (ChemSupply Australia, >99.5\%), 1-butanol (ChemSupply Australia, >99\%), 2-butanol (ChemSupply Australia, >99\%), acetic acid (Merk, >99\%), propanoic acid (ChemSupply Australia, >99\%), butanoic acid (ChemSupply Australia, >99\%), and methanol-d4 (Cambridge Isotope Laboratories, 99.8\%) were used as received.

Imbibed silica samples for $T_{1}-T_{2}$ analysis were prepared by soaking in excess liquid for at least 48 hours under ambient conditions. Silica particles were then separated from each liquid and rolled over a pre-soaked filter paper to remove any inter-particle liquid, with the imbibed silica spheres transferred to sealed $7 \mathrm{ml}$ glass vials for analysis. Each sample consisted of approximately $2.5 \mathrm{~g}$ of Q15, corresponding with around 160 particles; as such, each sample provided a well-averaged measurement of the surface-adsorbate interactions present between probe liquid and the model silica material employed. Sample preparation for methanol-d4 exchange analysis is described in Supplementary Note 2.

\section{$21 \quad$ NMR hardware}

$22{ }^{1} \mathrm{H}$ nuclear spin relaxation measurements were performed using an Oxford Instruments Geospec NMR 23 spectrometer equipped with a $B_{0}=0.3 \mathrm{~T}$ parallel plate permanent magnet array (providing a ${ }^{1} \mathrm{H}$ frequency of $\left.24 v_{0}=12.7 \mathrm{MHz}\right)$ and a $53 \mathrm{~mm}$ Q-sense probe. All measurements were performed at room temperature $(25 \pm$ $25 \quad 1^{\circ} \mathrm{C}$ ) and ambient pressure.

\section{$262 \mathrm{D}$ relaxation analysis}

$27 T_{1}-T_{2}$ relaxation correlation data was acquired by applying the $2 \mathrm{D}$ pulse sequence in Figure $1 \mathrm{a},{ }^{20}$ wherein $90^{\circ}$ and $180^{\circ}$ radio frequency (RF) pulses are represented by think and thick vertical bars, respectively. Following initial polarisation of the spin system along the direction of the static magnetic field $B_{0}$ (conventionally termed the $z$-axis), a $180^{\circ} \mathrm{RF}$ pulse inverts the sample magnetisation onto the $-\mathrm{z}$ axis. Here, 
characterised by the relaxation time constant $T_{1}$, and was encoded within the indirect dimension of our 2D relaxation data through the application of a variable recovery time $\tau$. The spin system is then rotated into the transverse plane via a $90^{\circ} \mathrm{RF}$ pulse, inducing transverse relaxation processes. A train of $n 180^{\circ}$ RF pulses induces $n$ spin echoes, which decay in magnitude due to $T_{2}$; each echo magnitude $S\left(n t_{e}, \tau\right)$ was recorded as a single data point (white data point in Figure 1a), defining the direct dimension of our relaxation data. The pulse sequence was then cycled to encode for $m$ different $\tau$ recovery times, forming an $(m \times n)$ data surface

(Figure 1d). The indirect dimension of our measurements was encoded by employing 16 logarithmically spaced $\tau$ values between $1 \mathrm{~ms}$ and $12 \mathrm{~s}$, while the direct dimension was encoded using $n=40,000$ echoes separated by an echo time of $t_{e}=100 \mu \mathrm{s}$. This short echo time, together with the low static magnetic field strength used here, was employed to mitigate undesired transverse relaxation phenomena resulting from magnetic susceptibility contrast effects at the solid-liquid interface. ${ }^{35}$ Measurements included 8 repeat scans separated by a recycle delay of $12 \mathrm{~s}\left(\gg 5 \times T_{1}\right.$ ), taking approximately 40 minutes per correlation measurement and resulting in signal-to-noise ratios of approximately 400.

$T_{1}-T_{2}$ relaxation correlation data may be described by a $2 \mathrm{D}$ Fredholm integral equation of the first kind: ${ }^{47}$

$$
\frac{S\left(n t_{e}, \tau\right)}{S(0, \infty)}=\iint K_{12}\left(n t_{e}, T_{2}, \tau, T_{1}\right) F\left(T_{1}, T_{2}\right) d \log _{10}\left(T_{1}\right) d \log _{10}\left(T_{2}\right)+\varepsilon\left(n t_{e}, \tau\right) .
$$

Here $S\left(n t_{e}\right) / S(0, \infty)$ is the normalised spin echo signal magnitude, while $\varepsilon\left(n t_{e}, \tau\right)$ represents the experimental noise, assumed Gaussian with zero mean. The kernel function $K_{12}\left(n t_{e}, T_{2}, \tau, T_{1}\right)$ describes the predicted form of $T_{1}$ and $T_{2}$ relaxation, which for the pulse sequence in Figure $1 \mathrm{a}$ is given by: ${ }^{20}$

$$
K_{12}\left(n t_{e}, T_{2}, \tau, T_{1}\right)=\left[1-2 \exp \left(\frac{-\tau}{T_{1}}\right)\right] \exp \left(\frac{-n t_{e}}{T_{2}}\right)
$$

Finally, the term $F\left(T_{1}, T_{2}\right)$ represents the targeted $2 \mathrm{D}$ distribution of $T_{1}$ and $T_{2}$ relaxation time constants. Distributions were obtained via a numerical inversion of each acquired relaxation data surface according to Equations (4) and (5). As this is an ill-posed problem in the presence of experimental noise, ${ }^{41}$ stability of the inverted distributions was achieved through the application of Tikhonov regularisation, ${ }^{68}$ with the degree of smoothing determined via the generalised cross-validation (GCV) method. ${ }^{69}$ Distributions were limited to $(200 \times 200)$ values, with each dimension bound within the range $\left\{10^{-4}, 10^{1}\right\} \mathrm{s}$ (note that correlation plots in

Figure 1 are shown with reduced bounds to increase correlation peak clarity). Inversions were performed using a 2D fast Laplace inversion algorithm written in Matlab, as first implemented by Mitchell et al. ${ }^{70}$

\section{$H / D$ exchange}

For the analysis of H/D exchange at the methanol-d4/silica interface, $T_{2}$ decay data was acquired by applying the standard Carr-Purcell Meiboom-Gill (CPMG) sequence. The magnitude of $n=40,000$ spin echoes were acquired separated by an echo time of $t_{e}=400 \mu \mathrm{s}$. Further details are provided in Supplementary Note 2. 


\section{Acknowledgements}

2 The authors thank Fuji Silysia (Japan) for providing the mesoporous silica and acknowledge funding from the

3 Australia Research Council (IC150100019 and LP1801001116).

\section{$5 \quad$ Additional information}

\section{Author Contributions}

7 N.R. and M.L.J. designed the research. N.R. performed the experiments and analysed the data. E.F.M and

8 M.L.J. supervised the research. N.R. and M.L.J wrote the manuscript. All authors discussed the results and 9 commented on the manuscript.

10 Supplementary Information Details

11 The Supplementary Information file contains:

- Supplementary Note 1: Surface Relaxation Theory

- Supplementary Note 2: Methanol-d4 Exchange Experiments

- Supplementary Table 1

- Supplementary Figures $1-3$

\section{Data Availability}

17 Associated data is available in the Supplementary Information file or from the corresponding author upon 18 reasonable request.

19 Competing Interests

20 The authors declare no competing interests. 


\section{References}

1. Zaera, F. Surface chemistry at the liquid/solid interface. Surface Science 605, 1141-1145 (2011).

2. Zaera, F. Probing liquid/solid interfaces at the molecular level. Chem. Rev. 112, 2920-2986 (2012).

3. Gladden, L. F. \& Mitchell, J. Measuring adsorption, diffusion and flow in chemical engineering: applications of magnetic resonance to porous media. New J. Phys. 13, 035001 (2011).

4. Kinn, B. E., Myers, T. R. \& Allgeier, A. M. Surface enhanced nuclear magnetic resonance relaxation mechanisms and their significance in chemical engineering applications. Curr. Opin. Chem. Eng. 24, 115-121 (2019).

5. Kowalewski, J. \& Mäler, L. Nuclear spin relaxation in liquids: theory, experiments, and applications. (CRC Press, 2017).

6. Korb, J.-P. Multiscale nuclear magnetic relaxation dispersion of complex liquids in bulk and confinement. Prog. Nucl. Magn. Reson. Spectrosc. 104, 12-55 (2018).

7. Brown, R. J. S. The Earth's-field NML development at Chevron. Concepts Magn. Reson. 13, 344-366 (2001).

8. Kleinberg, R. L. NMR well logging at Schlumberger. Concepts Magn. Reson. 13, 396-403 (2001).

9. Mitchell, J. \& Fordham, E. J. Contributed Review: Nuclear magnetic resonance core analysis at $0.3 \mathrm{~T}$. Rev. Sci. Instrum. 85, 111502 (2014).

10. Korb, J. P. NMR and nuclear spin relaxation of cement and concrete materials. Current Opinion in Colloid and Interface Science 14, 192-202 (2009).

11. Korb, J.-P. Nuclear magnetic relaxation of liquids in porous media. New J. Phys. 13, 035016 (2011).

12. D'Agostino, C. et al. Solvent inhibition in the liquid-phase catalytic oxidation of 1,4-butanediol: understanding the catalyst behaviour from NMR relaxation time measurements. Catal. Sci. Technol. 6, 7896-7901 (2016).

13. Isaacs, M. A. et al. A spatially orthogonal hierarchically porous acid-base catalyst for cascade and antagonistic reactions. Nat. Catal. 3, 921-931 (2020).

14. Filippini, G. et al. Light-driven, heterogeneous organocatalysts for C-C bond formation toward valuable perfluoroalkylated intermediates. Sci. Adv. 6, 9923-9934 (2020).

15. Robinson, N. et al. Low-field NMR relaxation-exchange measurements for the study of gas admission in microporous solids. Phys. Chem. Chem. Phys. 22, 13689-13697 (2020).

16. Chen, J. J. et al. ExSitu NMR relaxometry of metal-organic frameworks for rapid surface-area screening. Angew. Chemie - Int. Ed. 52, 12043-12046 (2013).

17. Song, Y. Q. Magnetic resonance of porous media (MRPM): A perspective. J. Magn. Reson. 229, 12-24 (2013).

18. Callaghan, P. T. et al. Recent Fourier and Laplace perspectives for multidimensional NMR in porous media. Magn. Reson. Imaging 25, 441-444 (2007). 
19. Bernin, D. \& Topgaard, D. NMR diffusion and relaxation correlation methods: New insights in heterogeneous materials. Curr. Opin. Colloid Interface Sci. 18, 166-172 (2013).

20. Song, Y.-Q. et al. T1-T2 Correlation Spectra Obtained Using a Fast Two-Dimensional Laplace Inversion. J. Magn. Reson. 154, 261-268 (2002).

21. Robinson, N., Robertson, C., Gladden, L. F., Jenkins, S. J. \& D’Agostino, C. Direct correlation between adsorption energetics and nuclear spin relaxation in a liquid-saturated catalyst material. ChemPhysChem 19, 2472-2479 (2018).

22. King, J. N., Lee, V. J., Ahola, S., Telkki, V. V. \& Meldrum, T. Ultrafast Multidimensional Laplace NMR Using a Single-Sided Magnet. Angew. Chemie - Int. Ed. 55, 5040-5043 (2016).

23. Washburn, K. E. \& Callaghan, P. T. Tracking Pore to Pore Exchange Using Relaxation Exchange Spectroscopy. Phys. Rev. Lett. 97, 175502 (2006).

24. Mitchell, J. et al. Validation of NMR relaxation exchange time measurements in porous media. J. Chem. Phys. 127, 234701 (2007).

25. Hürlimann, M. D. \& Venkataramanan, L. Quantitative measurement of two-dimensional distribution functions of diffusion and relaxation in grossly inhomogeneous fields. J. Magn. Reson. 157, 31-42 (2002).

26. Hürlimann, M. D., Venkataramanan, L. \& Flaum, C. The diffusion-spin relaxation time distribution function as an experimental probe to characterize fluid mixtures in porous media. J. Chem. Phys. 117, 10223-10232 (2002).

27. Ahola, S. et al. Ultrafast multidimensional Laplace NMR for a rapid and sensitive chemical analysis. Nat. Commun. 6, 1-7 (2015).

28. Mankinen, O. et al. Ultrafast diffusion exchange nuclear magnetic resonance. Nat. Commun. 11, 1-8 (2020).

29. Sun, B. \& Dunn, K. J. A global inversion method for multi-dimensional NMR logging. J. Magn. Reson. 172, 152-160 (2005).

30. Chandrasekera, T. C., Mitchell, J., Fordham, E. J., Gladden, L. F. \& Johns, M. L. Rapid encoding of T1 with spectral resolution in n-dimensional relaxation correlations. J. Magn. Reson. 194, 156-161 (2008).

31. Arns, C. H., Washburn, K. \& Callaghan, P. T. Multidimensional NMR inverse Laplace spectroscopy in porous media. Magn. Reson. Imaging 25, 548-549 (2007).

32. Zhang, Y., Xiao, L., Li, X. \& Liao, G. T1-D-T2 correlation of porous media with compressed sensing at low-field NMR. Magn. Reson. Imaging 56, 174-180 (2019).

33. Terenzi, C., Sederman, A. J., Mantle, M. D. \& Gladden, L. F. Spatially-resolved 1H NMR relaxationexchange measurements in heterogeneous media. J. Magn. Reson. 299, 101-108 (2019).

34. Vashaee, S. et al. Local T1-T2 distribution measurements in porous media. J. Magn. Reson. 287, 113122 (2018).

35. Mitchell, J., Chandrasekera, T. C., Johns, M. L., Gladden, L. F. \& Fordham, E. J. Nuclear magnetic resonance relaxation and diffusion in the presence of internal gradients: The effect of magnetic field 
strength. Phys. Rev. E 81, (2010).

36. Terenzi, C., Sederman, A. J., Mantle, M. D. \& Gladden, L. F. Enabling High Spectral Resolution of Liquid Mixtures in Porous Media by Antidiagonal Projections of Two-Dimensional $1 \mathrm{H}$ NMR COSY Spectra. J. Phys. Chem. Lett. 10, 5781-5785 (2019).

37. Fleury, M. \& Romero-Sarmiento, M. Characterization of shales using T1-T2 NMR maps. J. Pet. Sci. Eng. 137, 55-62 (2016).

38. Song, Y.-Q. \& Kausik, R. NMR application in unconventional shale reservoirs - A new porous media research frontier. Prog. Nucl. Magn. Reson. Spectrosc. 112-113, 17-33 (2019).

39. Weber, D., Mitchell, J., Mcgregor, J. \& Gladden, L. F. Comparing strengths of surface interactions for reactants and solvents in porous catalysts using Two-dimensional NMR relaxation correlations. J. Phys. Chem. C 113, 6610-6615 (2009).

40. Thomas, J. M. \& Thomas, W. J. Principles and practice of heterogeneous catalysis. Principles and practice of heterogeneous catalysis (Wiley-VCH, 2015).

41. Mitchell, J., Chandrasekera, T. C. \& Gladden, L. F. Numerical estimation of relaxation and diffusion distributions in two dimensions. Prog. Nucl. Magn. Reson. Spectrosc. 62, 34-50 (2012).

42. Brownstein, K. R. \& Tarr, C. E. Importance of classical diffusion in NMR studies of water in biological cells. Phys. Rev. A 19, 2446-2453 (1979).

43. Barrie, P. J. Characterization of porous media using NMR methods. Annual Reports on NMR Spectroscopy 41, 265-316 (2000).

44. Mitchell, J., Broche, L. M., Chandrasekera, T. C., Lurie, D. J. \& Gladden, L. F. Exploring Surface Interactions in Catalysts Using Low-Field Nuclear Magnetic Resonance. J. Phys. Chem. C 117, 1769917706 (2013).

45. Godefroy, S., Korb, J.-P., Fleury, M. \& Bryant, R. G. Surface nuclear magnetic relaxation and dynamics of water and oil in macroporous media. Phys. Rev. E 64, 021605 (2001).

46. D'Agostino, C., Mitchell, J., Mantle, M. D. \& Gladden, L. F. Interpretation of NMR Relaxation as a Tool for Characterising the Adsorption Strength of Liquids inside Porous Materials. Chem. - A Eur. J. 20, 13009-13015 (2014).

47. Venkataramanan, L., Yi-Qiao Song \& Hurlimann, M. D. Solving Fredholm integrals of the first kind with tensor product structure in 2 and 2.5 dimensions. IEEE Trans. Signal Process. 50, 1017-1026 (2002).

48. Stapf, S., Kimmich, R. \& Seitter, R. O. Proton and deuteron field-cycling NMR relaxometry of liquids in porous glasses: Evidence for Lévy-walk statistics. Phys. Rev. Lett. 75, 2855-2858 (1995).

49. D’Agostino, C., Mitchell, J., Gladden, L. F. \& Mantle, M. D. Hydrogen Bonding Network Disruption in Mesoporous Catalyst Supports Probed by PFG-NMR Diffusometry and NMR Relaxometry. J. Phys. Chem. C 116, 8975-8982 (2012).

50. Rottreau, T. J., Parlett, C. M. A., Lee, A. F. \& Evans, R. Diffusion NMR Characterization of Catalytic Silica Supports: A Tortuous Path. J. Phys. Chem. C 121, 16250-16256 (2017).

51. Robinson, N. \& D'Agostino, C. NMR Investigation into the Influence of Surface Interactions on Liquid 
Diffusion in a Mesoporous Catalyst Support. Top. Catal. 63, 319-327 (2020).

52. Aguilar, J. A., Nilsson, M., Bodenhausen, G. \& Morris, G. A. Spin echo NMR spectra without J modulation. Chem. Commun. 48, 811-813 (2012).

53. Robinson, N., Gladden, L. F. \& D'Agostino, C. Exploring catalyst passivation with NMR relaxation. Faraday Discuss. 204, 439-452 (2017).

54. Ward-Williams, J., Korb, J. P. \& Gladden, L. F. Insights into Functionality-Specific Adsorption Dynamics and Stable Reaction Intermediates Using Fast Field Cycling NMR. J. Phys. Chem. C 122, 20271-20278 (2018).

55. Ward-Williams, J. et al. Characterizing Solid-Liquid Interactions in a Mesoporous Catalyst Support Using Variable-Temperature Fast Field Cycling NMR. J. Phys. Chem. C 125, 8767-8778 (2021).

56. D'Agostino, C., Chansai, S., Gladden, L. F. \& Hardacre, C. Correlating the strength of reducing agent adsorption with $\mathrm{Ag} / \mathrm{Al} 2 \mathrm{O} 3$ catalyst performances in selective catalytic reduction (SCR) of NOx. Catal. Today (2021). doi:10.1016/j.cattod.2021.01.013

57. Roy, D. et al. Nonpolar adsorption at the silica/methanol interface: Surface mediated polarity and solvent density across a strongly associating solid/liquid boundary. J. Phys. Chem. C 117, 27052-27061 (2013).

58. Han, J. W., James, J. N. \& Sholl, D. S. First principles calculations of methylamine and methanol adsorption on hydroxylated quartz (0 00 1). Surf. Sci. 602, 2478-2485 (2008).

59. Ishikita, H. \& Saito, K. Proton transfer reactions and hydrogen-bond networks in protein environments. J. R. Soc. Interface 11, 20130518 (2014).

60. Lowe, B. M., Skylaris, C. K. \& Green, N. G. Acid-base dissociation mechanisms and energetics at the silica-water interface: An activationless process. J. Colloid Interface Sci. 451, 231-244 (2015).

61. Olivieri, G., Goel, A. \& Brown, M. A. Molecular level insight on the adsorption of carboxylic acids to oxide nanoparticles in aqueous solution by $\mathrm{X}$-ray photoelectron spectroscopy. Chem. Commun. 52, 9040-9043 (2016).

62. McDonald, P. J., Korb, J.-P., Mitchell, J. \& Monteilhet, L. Surface relaxation and chemical exchange in hydrating cement pastes: A two-dimensional NMR relaxation study. Phys. Rev. E 72, 011409 (2005).

63. Kimmich, R. Principles of soft-matter dynamics: Basic theories, non-invasive methods, mesoscopic aspects. Principles of Soft-Matter Dynamics: Basic Theories, Non-invasive Methods, Mesoscopic Aspects (Springer Netherlands, 2012). doi:10.1007/978-94-007-5536-9

64. Van Landeghem, M., Haber, A., D'espinose De Lacaillerie, J.-B. \& Blümich, B. Analysis of multisite 2D relaxation exchange NMR. Concepts Magn. Reson. Part A 36A, 153-169 (2010).

65. Krzyżak, A. T. \& Habina, I. Low field 1H NMR characterization of mesoporous silica MCM-41 and SBA15 filled with different amount of water. Microporous Mesoporous Mater. 231, 230-239 (2016).

66. Gilli, P., Bertolasi, V., Pretto, L. \& Gilli, G. Outline of a transition-state hydrogen-bond theory. J. Mol. Struct. 790, 40-49 (2006).

67. Gilli, P., Pretto, L., Bertolasi, V. \& Gilli, G. Predicting Hydrogen-Bond strengths from Acid-Base molecular 
properties. the pKa slide rule: Toward the solution of a Long-Lasting problem. Acc. Chem. Res. 42, 3344 (2009).

3 68. Tikhonov, A. N. \& Arsenin, V. I. A. Solutions of ill-posed problems. (SIAM, 1977).

4 69. Golub, G. H., Heath, M. \& Wahba, G. Generalized cross-validation as a method for choosing a good ridge parameter. Technometrics 21, 215-223 (1979).

6

70. Mitchell, J. et al. Determining NMR flow propagator moments in porous rocks without the influence of relaxation. J. Magn. Reson. 193, 218-225 (2008). 